\title{
Business Intelligence Design Model (BIDM) for University
}

\author{
Budour Ahmed Al Farsi \\ Faculty of Computing and Information Technology \\ Sohar University \\ Sultanate of Oman
}

\author{
Dinesh Kumar Saini \\ Faculty of Computing and Information Technology \\ Sohar University \\ Sultanate of Oman \\ Research Fellow and Adjunct Faculty, University of \\ Queensland Australia
}

\begin{abstract}
Information and knowledge are the backbone of any organization's development because they are used in decision making processes. In addition, the management level of any organization is looking forward to ensure the quality and the effectiveness of the work performances depending on the knowledge to make the right decisions. But by the increased amount of information produced by the organizations the decision making processes becomes more complex and needs lots of time to be taken. Therefore, they have to upgrade their work and the way of decision making processes by using new technologies like the Business Intelligence (BI). BI can help the organizations to make the best use of information and creating a knowledge base which supports the decision making processes. Therefore, the universities as any organizations are looking forward to improve their work performances to keep pace with the world's development and manage the increase of the volume of the information needed for the decision making process. Therefore, this research paper is to introduce a Business Intelligence Design Model (BIDM). BIDM is a model that is going to help university to use the business intelligence systems to produce the reports which achieve the accuracy, reliability and the effectiveness to support the decision making processes.
\end{abstract}

\section{General Terms}

Information Systems, Business Intelligence, Data Engineering

\section{Keywords}

Business Systems, Business Intelligence, Operational Data,

\section{INTRODUCTION}

Business Intelligence (BI) refers to the technology collection which helps a business, to acquire a better decision quality by using data, covering the process of extracting information and knowledge from a broad catalog of data. Business Intelligence (BI) [1] provides the ability to analyze business information in order to support and improve decision making at the management level. Therefore, any organization looking to become a market leader needs to embrace continuous improvement and innovation, and this requires the adoption of increasingly more advanced approaches to BI. In addition, the development of the businesses these days and the increased data and information needed for the daily operations are very worth-full to the business strategies. The universities as any other organizations are looking forward to speed up the development processes to keep pace with the new technologies development. So that, Business Intelligence (BI) is one of the new technologies which can leads to the development of the businesses.
Business Intelligence (BI) is the way of combining the operational data with the analytical tools to present complex and competitive information to planners and decision makers by producing informative and meaningful reports. BI is defined using many ways by the researchers. It is defined as the systems that provide the ability to analyze business information in order to support and improve management decision making across a broad range of business activities [1]. It will make a best use of information and knowledge by improving the knowledge management advances. In addition, it provides the information needed by the management to take the right decisions for the right time because taking decisions is difficult and long process which will cause an effect to the university as an important educational institution in Oman. Therefore, there will is a need for using the Business Intelligence to support the way of processing and making a decisions at the university. These tools are going to be used through a design model that will allows them to enter the data and produce reports that will support the decisions made and helps in forecasting for the extended years.

\section{BACKGROUND WORK}

Business Intelligence (BI) has become a new fashion in information systems (IS) since 2000s. Therefore, BI is used to make better management decisions in organizations. It is described to help users to make better decisions by technologies, applications, and processes for gathering, storing, accessing and analyzing data. Therefore, to make a research for Business Intelligence (BI) solution there will be a need to search for literature reviews that can support the way of proofing the concept and the decision for BI usage.

\subsection{Literature Review Case Studies}

The business Intelligence systems are implemented for many sides and some of the examples are studied in detail in this paper. Some of the universities have already implemented Business intelligent systems and efforts are made to review and analyze these universities in this paper.

Business Intelligence Financial Information Systems for some Organizations

It has been used as implementation of Business Intelligence Financial Information Systems (FIS) [2] for organizations listed on stock exchange. It can assess the intelligence of Financial Information Systems to achieve enhanced decision support in Iranian organizations listed on stock exchange. It is used to gain competitive advantage through integrating decision support into the environment of these systems. The Business intelligence of the Financial Information System is used to track the financial data and generates reports with alerts that assist managers in effectively running their business processes using limited resources after analyzing the financial 
data they gain. Business Intelligence (BI) [2] was considered to be an instrument of analysis, providing automated decisionmaking about the business processes. It uses data-warehouse analysis with data mining and online analysis processing (OLAP). It integrates the analysis of data with decision analysis tools to provide the right information to the right persons throughout the organization with the purpose of improving strategic and tactical decisions.

\subsubsection{Business Intelligence models at university of Quebec}

In the other hand, Business Intelligence models are also used in universities like University of Quebec. As a modern universities [3] uses a business processes model that makes a collection of business process and the existing knowledge and experiences to be documented using standardized method and models. The typical purposes [3] of these documentations are to document the relationship between the business processes and IT, to have a common reference for change and process improvement and to ensure consistent quality in process execution. Svenssona and Hvolbyb [3] identifying the university of Quebec has establishing the objectives and monitoring the performances through the reference model to ensure the relevant processes and performances. So, they define the purposes of the business process reference model for the universities are to:

$>$ Provide access to a reference framework of the university business process.

$>$ Link the performance of the universities with the global business processes.

$>$ Link the IT systems and the business functions.

$>$ Identify the main elements needed for the performing the needed functions.

Therefore, the reference model of the universities aimed to improve the process quality and reducing the efforts and the time consumed in collecting the reports of the data needed. In addition, the reference model can help the universities to establish a new infrastructure in building modern universities.

\subsubsection{Business Intelligence at Indiana University}

Business Intelligence (BI) at Indiana University (IU) [4] is used as a set technologies and processes that help decision makers to understand the analyzed data of the organizational performance. The purposes of the BI at IU are to:

$>$ Integrate information across applications, campuses, and external partners

$>$ Report all the information consistently through all levels

$>$ Provide specific information to take key decision making

$>$ Increase the competitions with other institutions and organizations

The UI have many projects based on BI like the BI Tool Evaluation. Its objective is to evaluate a variety of BI reporting tools based on a wide range of selection criteria and recommend a set of BI tools which will meet the needs and be supported on an institutional-wide basis. Each tool classified according to their fundamental capabilities including Ad hoc, Reporting, Analytics, Dashboards, OLAP and report development.

\section{EVALUATION TOOLS}

Nowadays, information and knowledge represent the fundamental wealth of an organization. Enterprises try to utilize this wealth to gain competitive advantage when making important decisions [5]. They use the enterprise systems to converts and store the data in a database. Then they can use a pool of data to support the decisions and explore the needed knowledge. Therefore, they integrate decision support into the environment of their enterprise and work systems. In addition, they embedded the Business Intelligence (BI) in these enterprise systems to obtain the competitive advantage.

The objectives [5] of evaluation tools for BI are to see the effect of BI evaluation of enterprise systems on the decisionsupport environments of organizations. In addition, BI tools used to determine the main factors in the evaluation of $\mathrm{BI}$ competencies and their importance. The most important factors that can affect the evaluation tool can become through labelling. These factors [5] named as the following:

$>$ Analytical and Intelligent Decision-support

$>$ Providing Related Experiment and Integration with Environmental Information

$>$ Optimization and Recommended Model

$>$ Reasoning

$>$ Enhanced Decision-making Tools

$>$ Stakeholders

The evaluation tool is implemented in a VB.NET environment [5] and has three functionalities: To evaluate a new system, to edit/view and compare the evaluated system. The tool requests for [5] the system type, the organization type, the overall specifications of the enterprise systems and the specification of the organization. Then computations are done based on the factors and their loading. Reports which include the overall level of BI and its intelligence level in the six areas are depicted on the dashboards [5]. In addition, charts show a comparison of the factors that share in the intelligence of enterprise systems. The following figure 3.1 illustrates a sample of the intelligence-level of a sample system and its comparisons.

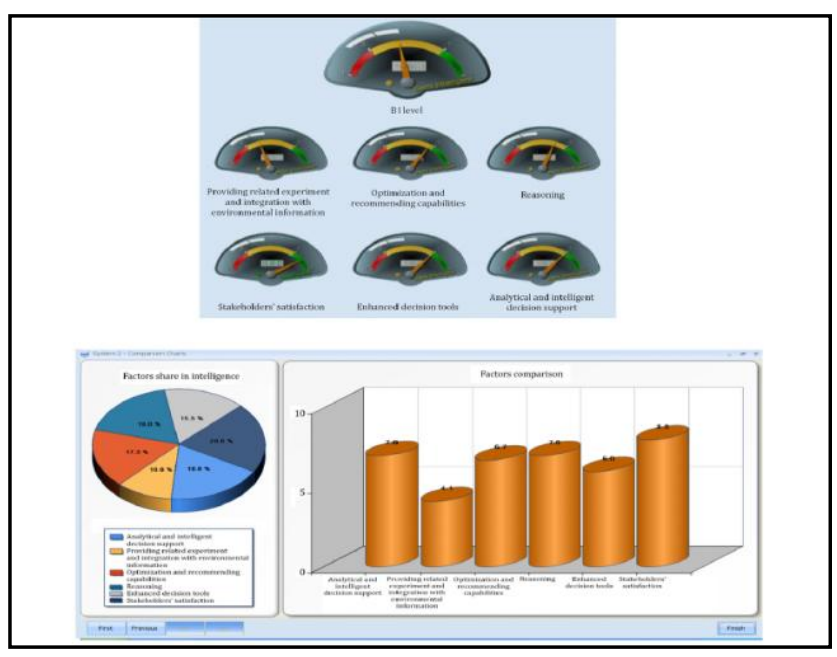

Figure 3.1 Intelligence dashboards and comparison charts of the designed tool [5] 
The Business intelligence at University

In the reality of making good decisions, the academic institutions are looking for the evidences and the information that supports the decisions made and the resources available for new decisions. Therefore, the researchers at University are looking for Business intelligence (BI) as a strategy to increase the quality, accessibility and the use of evidence. They considered the elements appeared in figure 3.2 as the foundation of the Business Intelligence (Radcliffe 2010).

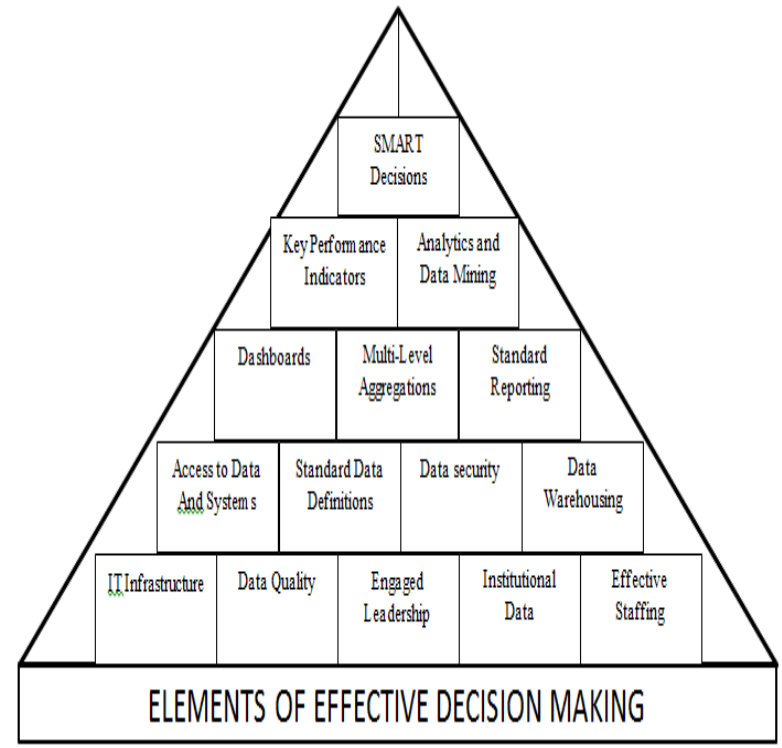

Figure 3.2 the Foundation of the Business Intelligence

Business Intelligence Initiative [6] is divided in two parts which are; People and Processes and Tools and Technology. The people and Processes contain Data governance, Forum for collaboration and sharing and training and development using the tool, analysis and processes improvement. The second part which is the Tools and technology include Oracle Business Intelligence Enterprise Edition (OBIEE), Dashboards, Analytics and BI publisher.

The aim of using the BI is to help for student recruitments and retention, course scheduling and support, purchasing and the employee satisfaction. In addition, it aims to control the increment of the costs, to increase the innovation and improve the efficiency and productivity. Therefore, they use the reporting tools to empowering the faculty and staff to access the needed data.

Figure 3.3 shows the plan for the implementation of the BI tools which include the organization supporting collaboration that contains:

$>$ Business Intelligence Steering Committee that provide overall direction and coordination

$>$ Data governance committee that connect the data custodians and other actors responsible for data integrity

> University of Minnesota Analytics Collaborative which is A virtual organization serving as a "Business intelligence competency centre" to guide and promote training, improve metadata and facilitate innovation, sharing and cooperation.

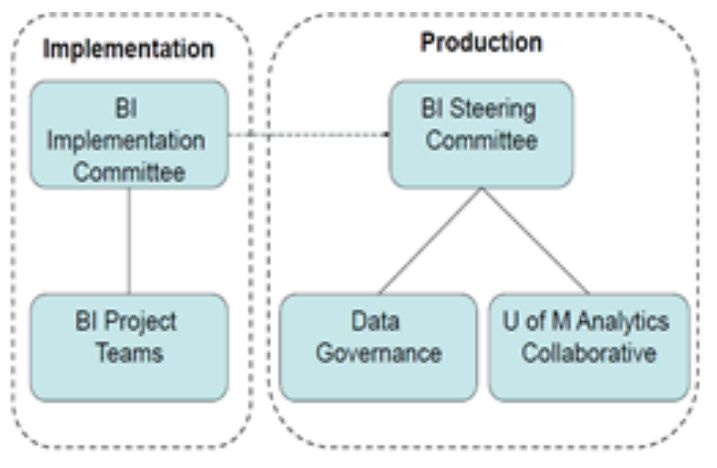

Figur3.3 Implementation of BI

As business Intelligence they use new tools because The oracle BI toolset [6] gives the capability that are harder to be produced and the easier to be used and improving the accessibility of the data.

\section{FINDINGS}

The case studies in the literature reviews are mentioned for the organizations that uses BI for several aims. The common purpose of the literature reviews is to ensure consistent quality in process execution and the quality of data for making decisions. In addition, they aimed to support the managers to take the right decisions through running the businesses more frequently. Also, by using Business intelligence the managers will link the performance of their organizations with global business processes and the IT infrastructure.

\section{METHODOLOGY}

Research methodology [6] is the way of the investigation and research for practical problems to increase the knowledge. It is the way of collecting the data and information needed to define better ways for improvement. Also, Business Intelligence is a way that supports the understanding the internal environments of the university and making convenient decisions for its woks improvements. These improvements can affect the business by giving move values to their processes. Therefore, the business intelligence design model as a development for the university's current status decisions there is a need to define the main issues for the usage of the model and the best way it is going to work with. In addition, using the research methodology is beneficial to define the weaknesses of the current way of decision making and define the best way to recover from them.

This research uses the research methodology techniques for gathering the data like literature reviews, personal interviews. Literature reviews [7] are a form of research that represents the knowledge of the selected research area and helps the researcher to look in depth in the research and a voiding the repeated issues with the other researches. So, it is used as a research proof to the selected area.

In the other hand, the quantitative methodology is used to explain the current phenomena and justify the current situations. It could be done through documents, mails, group interviews or individual interviews.

Personal interviews include unstructured, open end questions. They provide more subjective data than surveys because they include the intended user and their needs with the experiments and their knowledge added to the model's values. Therefore, they help to uncover the issues that can occur with the new product and encourage the research to meet the main aims and objectives of it. 
The interview was done with an experiment of the Information Technology Services (ITS) at the university to define the necessary information related to the Business Intelligence (BI) and the ways of extracting the data from the system. The questions was asked as open ended questions which focused in the way of collecting data to the system and the process of identifying the needed information for the decisions and the challenges faced during the decision making processes. The second interview was with the head of the Computing department. The interview was close ended questions about the needed of the management decisions and the expectation from the development for the new technology which is Business Intelligence Design Model (BIDM).

Both interviews give realistic information about the way of taking decisions and the level of interaction between the management levels and the information provided. They depend in more than one source to produce knowledgeable reports to make decisions which take lot of time and spend lots of costs. In addition, they face some challenges that cause many problems with the traditional ways because of the different sources and the huge volume of data available to be analyzed for decision making processes. Therefore, he tells that there will be a need for this research because of the huge volume of data available for analysing for decision making processes and the challenges faced to make reliable and accurate decisions. In addition, there is a need for high degree of the quality of data by avoiding the data reputation or data lost and to keep pace of the growth of the Information Technology (IT). Also, the reports generated manually needs huge amount of cost and spends a lot of time and that can cause latency of decisions made. Furthermore, the collected data from the interviews the need for that research project to depend in extracting high quality of data regarding to the increased number of students and staff and help in making reliable information by see the processes of the work performances and forecast for the future.

\section{BUSINESS INTELLIGENCE TECHNIQUES}

By the increment of the standards, automation and technologies that has been done in modern business and the huge amount of data produced, the decision making process has become complicated [1].

The usage of the information and knowledge is the main ways to make decisions especially for large organizations. In addition, the top level management at any organization is looking forward to ensure the quality and the effectiveness of the decisions they made depending on the information and knowledge produced in the daily operations to keep pace of the development around the world. Therefore, Business intelligence has applied the functionality, scalability, and reliability of modern database management systems to build ever-large data warehouses, and to utilize data mining techniques to extract business advantage from the vast amount of available enterprise data [2].

Therefore, according to the evolution of the business intelligence applications and services of Business intelligence, this paper represents a review of BI techniques used through the time period starting from 1990 to 2015. Business Intelligence have been used by the organizations for many years to monitor, report on, analyze, and improve the performances of their business operations.

These organizations start with applications to simplify the process of decision making processes. The applications used for BI are spreadsheets, Oracle databases, oracle data mining and data warehousing as the following:

\subsection{Spreadsheets}

Spreadsheet is one of the most important applications that are used for BI. Therefore, it has a sharp increase of usage instead of the other technologies.

The excel application is one of the most important spreadsheets applications used for BI that its first release on 1987. It has been developed by Microsoft for Microsoft Windows and Mac OS. It is used for calculation, graphing tools, pivot tables and macro programming language called Visual Basic for Applications. It has been a very widely applied spreadsheet for these platforms, especially since version 5 in 1993, and it has replaced lotus 1-2-3 as the industry standard for spreadsheets. Excel forms part of Microsoft Office. Excel 2.0 is the first version of Excel for Intel platform which include a run-time version of windows. Then excel 3.0 has been released on 1990 that include toolbars, drawing capabilities, outlining, add-in support, 3D charts and others. Then excel 4.0 which include the Easter egg that reveals a hidden animation of a dancing set of numbers 1 through 3 that represent Lotus 1-2-3. Then version 5.0 that has been includes Visual basic Application (VBA) that allows the creation of forms and in-worksheet controls to communicate with the user. The versions 5.0 to 9.0 of the Excel contain various Easter eggs. Then the other versions has include enhancements and included in the office with new tools available within the current office available with. Therefore, spreadsheet server requires components like the application component, Microsoft .Net framework and Microsoft Visual Studio Tools for office. Figure 4.1 introduce the chart for the versions released of spreadsheets.

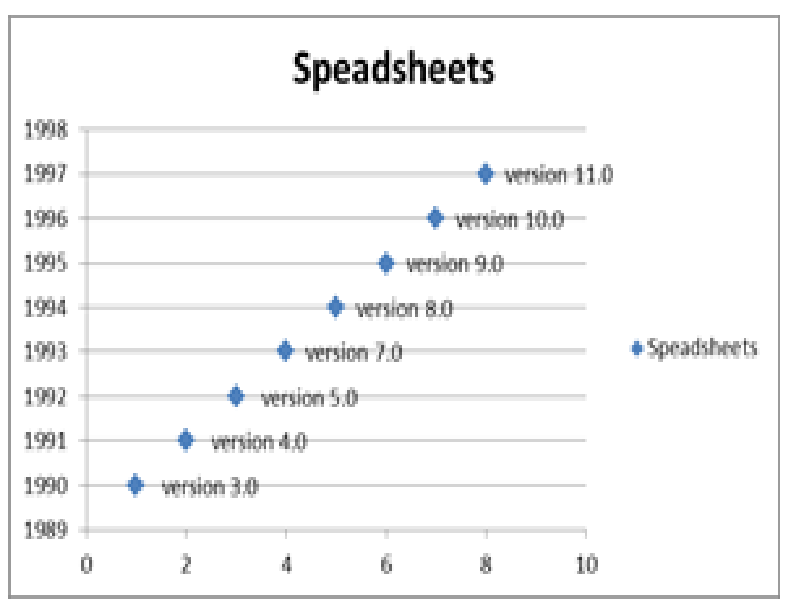

Figure 4.1 the released versions of spreadsheets

\subsection{Databases}

Oracle Business Intelligence Applications are built to provide universal adapters to extract, transform, and load (ETL) data from multiple source systems to be integrated across multiple Oracle Business Intelligence Applications to give a single view across the organization. In addition, oracle database is one of the relational database techniques that is used as data analytics [3]. Oracle databases deliver all the functionalities of analytic engines with scalability, security and reliability. Therefore, it has been upgraded and updated regarding to the requirement needed by the enterprises. Figure 4.2 introduce the chart for the oracle databases versions. 


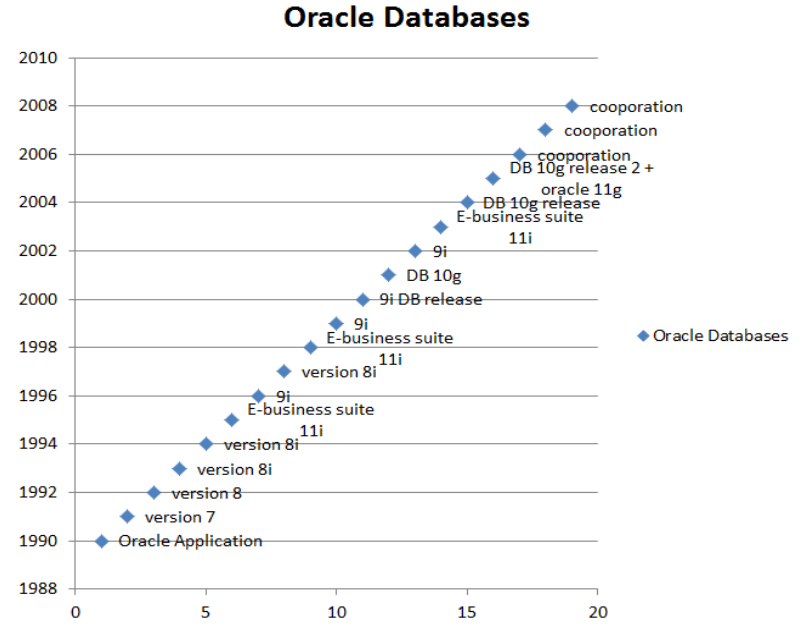

Figure 4.2 the released versions of Oracle Databases

\subsection{Data mining}

Researchers and scientists are proposing and founding theoretical foundations for data mining. Several interesting proposals have appeared in the past based various techniques like data reduction, data compression, probability and statistics theory, microeconomic theory, and pattern discovery-based inductive databases. Oracle implements a variety of data mining algorithms inside the oracle relational database. These implementations are integrated into oracle databases and processed directly on the data that are stored in the relational database tables. It provides the ability to manage the models securely and efficiently executes large volumes of data using SQL queries. The ODM system is organized around a few generic operations for example create, apply, test and manipulate data mining models by providing a general unified interface for data mining functions. The models are created and stored as database objects and the management is done within the database.

Oracle Data Mining released at the beginning in 2002 and named related to the Oracle database.

Business intelligence tools are the necessary conditions for applications of data mining, an efficient tool for getting new information from large datasets [4]. Figure 4.3 introduce the released versions of oracle data mining through the time.

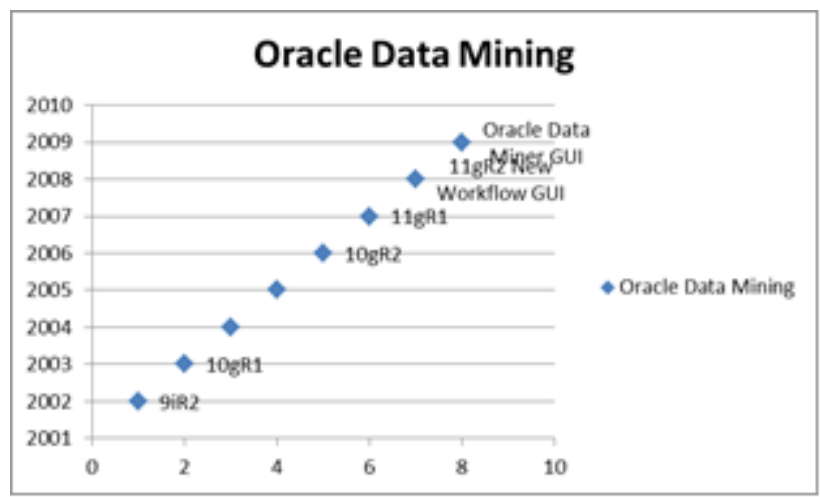

Figure 4.3 the released versions of oracle data mining

\subsection{Data warehousing}

A data warehouse is a relational database that is designed for query and analysis rather than for transaction processing. It contains historical data derived from transaction data, but it can include data from other sources. It separates analysis workload from transaction workload and enables an organization to consolidate data from several sources. In addition, a data warehouse environment includes an extraction, transportation, transformation and loading (ETL) solution, an online analytical processing (OLAP) engine, client analysis tools, and other applications that manage the process of gathering data and delivering it to business users.

\section{PROPOSED SOLUTIONS}

This paper is about Business Intelligence design model (BIDM) that is going to help the university to use the Oracle Business Intelligence tools to produce summary reports as the result to be depend on to take reliable and accurate decisions regarding to the need of the fast development and to keep pace with evolutions of the new technologies used around the world by upgrading their usage to new technologies that will support the decision making. In addition, it will help the university to identify problems and find solutions easily with limited times and keep track of the performances of the work done by the students and staff by monitor their processes. The objectives are to improve the knowledge management advances business by making the best use of information and make the data as data driven that will be reflected to the university's work performances and its behaviour regarding to the effects of the resulted reports. In addition, it allows accessing and analysing the data and information easily that supports the decision making process and information sharing in the complex environments.

The purpose of the Business Intelligence Design Model is to:

$>$ Improve the Knowledge management advances and make the best use of information.

$>$ Monitor the work performance of the university.

$>$ Ensure the reliability of the decisions made depending on the resulted reports.

$>$ The Business Intelligence design model is implemented using the Oracle Business Intelligence Enterprise Edition (OBIEE). Oracle Business Intelligence Enterprise Edition is a comprehensive suite of enterprise BI products that delivers a full range of analysis and reporting capabilities [8]. It gives the users the ability to perform analysis reports from detailed business data and provide real information for making critical decisions. It has two main components which are:

$>$ Oracle BI Presentation Services [8] that consist of interfaces which provides answers to business questions, display reports that contain content specific to the needs of individuals and groups and creating Oracle Business Intelligence Alerts based on analytics results.

Oracle Business Intelligence Applications [8] that are integrated with the operational applications to provide business metrics in reports, in context with an organizations business function and industry. In addition, Oracle Business Intelligence Applications include Extract Transform Load (ETL) routines to extract, transform, and load data into the Oracle Business Analytics Warehouse [8].

The OBIEE is selected because it allows a high degree of control over access to elements in Oracle BI applications. The security mechanism comprises Business logic object security, Presentation Catalogue object security and data level security [8]. In addition, it provides lots of features such as easy to use ad hoc query analysis, comprehensive suite of data visualizations, pixel perfect enterprise reporting, has the 
ability to act on insight by invoking business processes from within BI and Common Enterprise Information Model.

The Business intelligence design model (BIDM) that is demonstrated in figure 5.2 is the model that allows the university to get the reports depending of the realistic information provided. The model components as showed in figure 5.1 are; the data source or the data warehouse, the tools and applications component, the reports and the end users. Also, figure 5 describes the interactions of the model components. So, the user can use the BI publisher tools provided in Oracle Business Intelligence application to produce reports. First of all, the information are stored in different data sources and it contains a huge volume of data therefore the data can be stored inside a data warehouse. It uses the data warehouse as a source of the data because the data ware house [9] as a relational database is designed for query and analysis and contains the historical data derived from transaction data in addition to the other source's data. Also the data warehouse separate the analysis of the workload from the transactions workload that can facilitate the maintenance of the historical records and support the better understanding and usage of the data at the university. This data warehouse use Oracle $11 \mathrm{~g}$ as database application. So, the data needed by the OBIEE can be provided from the data source. Then, after using the OBIEE the end user can see the resulted reports to support the decision making processes. In addition, these resulted reports can be considered as knowledge and stored again in the data source or the data warehouse. So, these knowledge data or the resulted reports can be used again to supports the long term decisions.

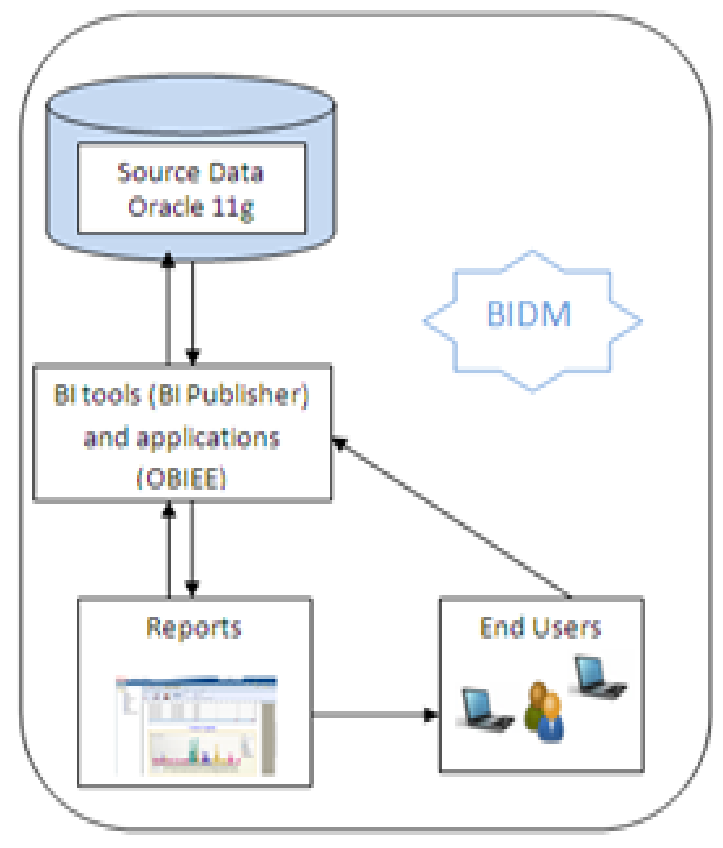

Figure 5.2 the structure of BIDM

\section{FUTURE DIRECTION}

The BIDM is tested after the installation that can produce reports with the sample data it has and can be implemented as suggested for the university. Thus, future work is to implement the BIDM research project at the university to satisfy the data and information requirements and to facilitate the decision making process. Moreover, the implementation of the research project will also require making training courses for the users before used to help them in using the new research project and facilitate the way of using it.
Furthermore, future work is making further studies of the BI as the evolution of the business and IT around the world over the time to use the BI as a decision maker and forecasting tool.

\section{CONCLUSION}

Nowadays, Business Intelligence (BI) has become a key technology of the information system for any organization. There are many reasons that affects the decision making processes such as the increased volume of data and information needed, the expandability of the university, the changes occurred in rules and the plans of the university and others. Therefore, the University as any organization gathers the information produced during the work timing to assess the business environments by supporting the decisions made by the management. Thus, university needs to keep pace with the modern technologies and upgrading the existing once. Therefore, Business Intelligence (BI) is one of the latest ways that can be used to gain the competitive advantages and cope with the changes and enlargement of the university. BI is aimed to help people to make accurate decisions using relevant information at the right time. In addition, BI can help to make accurate forecasts and plan strategies.

Therefore, this research demonstrates a design model that can help the university to use the BI as a way to support the decision making processes. The design model BIDM takes the data and the information from the data warehouse as a source because the data warehouse extract transform and load all the information at the university and use oracle $11 \mathrm{~g}$ as database application. So, it improves the knowledge management by making the best use of information. In addition, the Interaction of the user can be done through the BI publisher tools provided by OBIEE application. By using the OBIEE and BI publisher tools the user can see the report that supports the process of the decision making. Therefore, the resulted reports give new insight through the knowledge and producing more facilities that can be reflected on the university's work performances.

On the other hand, this report demonstrates the suggested BIDM by discussing the related work that has been done and the objective of then. In addition, it explains the research design chapter that include the research methodology and the analysis of them. Also, the implementation part examines one of the products that support the decision making processes and the way of producing report for the user.

\section{REFERENCES}

[1] P. A. Collier, E. Mohamed Z. and D. Michael J., "Meassuring the effects of the business intelligence systems: The relationship between business process andorganizational performance," International Journal of Accounting Information Systems, vol. 9, no. 3, pp. 135153, 2008.

[2] S. Rouhani and M. Mohmodian, "Financial Information Systems: Business Intelligence Perspectives," Journal of Advanced Computer Science and Technology Research, vol. 2, no. 2, pp. 66-78, 2012.

[3] C. Svenssona and H.-H. Hvolbyb, "Establishing a business process refernce model for universities," in CENTERIS 2012 - HCIST 2012, Algarve, Portugal, 2012.

[4] T. T. o. I. University, "Business Intelligence at Indiana University," 2013. [Online]. Available: 
http://www.indiana.edu/ iubi/index.shtml. [Accessed 17 06 2013].

[5] M. Ghazanfari, M. Jafari and S. Rouhani*, "A tool to evaluate the business intelligence of enterprise systems," Scientia Iranica E, vol. 18, no. 6, pp. 1579-1590, 2011.

[6] P. M. Radcliffe, "Business Intelligence at the University of Minnesota," Internal Communications Network, pp. 130, 16 December 2010.

[7] P. M. Radcliffe, "Business Intelligence at the University of Minnesota," Internal Communications Network, pp. 130, 16 December 2010.

[8] A. Ta'a, M. S. Abu Bakar and A. R. Saleh, "ACADEMIC BUSINESS INTELLIGENCE SYSTEM DEVELOPMENT USING SAS® TOOLS," SAS Global Forum 2008 (Workshop on Data Collection System for PHLI-MOHE), vol. 058, no. 058-2008, pp. 1-13, December 2008.

[9] V. Milutinovic, "A research methodology in the field of computer engineering for VLSI," in Microelectronics, 1995. Proceedings., 1995 20th International Conference, Serbia, 1995.

[10] Wikipedia, "Literature review," 05 August 2013. [Online]. Available: http://en.wikipedia.org/wiki/Literature_review. [Accessed 15 August 2013].
[11] R. Fuze, "OBIEE Knowledge Center," 2010. [Online]. Available:

http://www.ealliancebusinessintelligence.com/obiee_intr oduction.php. [Accessed 2507 2013].

[12] P. Lane, "Oracle Database Data warehouse," September 2007 [Online].

Available: http://docs.oracle.com/cd/B28359_01/server.111/b28313. pdf. [Accessed 0509 2013].

[13] T. Gang, K. Cui and S. Bei, "The research \& application of Business Intelligence system in retail industry," in IEEE International Conference on Automation and Logistics, Qingdao, 2008.

[14] W. Cody, J. Kreulen and W. Spangler, "The integration of business intelligence and knowledge management," IBM Research Division, Almaden Research Center, 650 Harry Road, San Jose, California 95120, USA , vol. 41, no. 4, pp. 697 - 713, 2010.

[15] C. Ping- Tsai and C. S. H., "On data integration and data mining for developing business intelligence," Systems, Applications and Technology Conference (LISAT), 2013 IEEE Long Island, pp. 1 - 6, 2013.

[16] M. Tvrdikova, "Support of Decision Making by Business Intelligence Tools," in Computer Information Systems and Industrial Management Applications, Minneapolis, 28-30 June 2007. 